OPEN ACCESS

Edited by:

Stefano Restaino,

Ospedale Santa Maria della

Misericordia di Udine, Italy

Reviewed by:

Raffaella Ergasti,

Catholic University of the Sacred

Heart, Italy

Matteo Loverro,

Agostino Gemelli University

Polyclinic, Italy

*Correspondence:

Song Gao

gaosong_sj@163.com

Specialty section:

This article was submitted to Gynecological Oncology,

a section of the journal

Frontiers in Oncology

Received: 17 February 2021 Accepted: 15 March 2021

Published: 31 March 2021

Citation:

Yan L, He Z, Li W, Liu N and Gao S (2021) The Overexpression of AcylCoA Medium-Chain Synthetase-3 (ACSM3) Suppresses the Ovarian

Cancer Progression via the Inhibition of Integrin B1/AKT Signaling Pathway.

Front. Oncol. 11:644840.

doi: 10.3389/fonc.2021.644840

\section{The Overexpression of Acyl-CoA Medium-Chain Synthetase-3 (ACSM3) Suppresses the Ovarian Cancer Progression via the Inhibition of Integrin $\beta 1 /$ AKT Signaling Pathway}

\author{
Limei Yan, Zeping He, Wei Li, Ning Liu and Song Gao* \\ Department of Obstetrics and Gynecology, Shengjing Hospital of China Medical University, Shenyang, China
}

Ovarian cancer is considered as one of the most fatal gynecologic malignancies. This work aimed to explore the effects and regulatory mechanism of Acyl-CoA medium-chain synthetase-3 (ACSM3, a subunit of CoA ligases) in ovarian cancer progression. As well as employing CCK-8 assay, clone formation assay, and cell cycle analysis were carried out to investigate cell proliferation ability. Wound healing assay and transwell assay were subsequently used to assess cell migration and invasion. Mice xenografts were then conducted to measure the effects of ACSM3 on tumor development in vivo. Our bioinformatics analysis suggested that the expression of ACSM3 was down-regulated in ovarian cancer tissues, and the low expression level of ACSM3 might related with poorer overall survival than high mRNA expression of ACSM3 in ovarian cancer patients. We artificially regulated the expression of ACSM3 to evaluate its effects on ovarian cancer malignant phenotypes. Our data revealed that the overexpression of ACSM3 inhibited cell proliferation, migration, and invasion of ovarian cancer cells. In contrast, the knock-down of $A C S M 3$ received the opposite results. Our western blot results showed that the Integrin $\beta 1 / A K T$ signaling pathway was negatively regulated by ACSM3 expression. Moreover, ACSM3 overexpression-induced suppression of cell migration and invasion activities were abolished by the overexpression of ITG $\beta 1$ (Integrin $\beta 1$ ). Additionally, the growth of ovarian cancer xenograft tumors was also repressed by the overexpression of ACSM3. And ACSM3 interference obtained the contrary effects in vivo. In summary, ACSM3 acts as a tumor suppressor gene and may be a potential therapeutic target of ovarian cancer.

Keywords: ovarian cancer, acyl-CoA medium-chain synthetase-3, integrin $\beta 1$, proliferation, metastasis

\section{INTRODUCTION}

Ovarian cancer is considered one of the most fatal gynecologic malignancies (1). Due to the asymptomatic development, ovarian cancer is frequently not diagnosed until at an advanced and incurable stage, which is seen as a silent killer (2). When ovarian cancer is diagnosed at an early stage that grows in one or two sides of ovaries, the cure rate of it could reach $90 \%$ (3). However, the 
cure rate of ovarian cancer attenuates substantially with the metastasis of the tumors to the uterus and bladder (stage II), the peritoneal cavity (stage III), or the visceral organs (stage IV) (3). More than $70 \%$ of patients with ovarian cancer are not diagnosed until tumors are actively metastasized and developed to stage III or IV (4). Unlike hematogenous disseminating cancers, ovarian cancer metastasizes throughout the peritoneal cavity by peritoneal fluid instead of vasculature (5). Then, ovarian cancer tumors compress the visceral organs and even spread to the parenchyma of the lung or liver (5). The metastasis and chemo-resistance cause the death of ovarian cancer patients (6). Despite abundant research on the pathogenesis and therapy for ovarian cancers, there is still a lack of authoritative treatment. Therefore, it is essential to find new potential target for effectual therapy in ovarian cancer.

Acyl-CoA medium-chain synthetase-3 (ACSM3) is a subunit of CoA ligases that plays a significant role in the progression of many diseases. Ruan et al. (7) reported that ACSM3 had a downregulated expression in hepatocellular carcinoma, which enhanced the metastasis via the AKT-WKN1 signaling pathway. Zhu et al. (8) confirmed that the loss of ACSM3 was associated with poor prognosis and immune exclusion in malignant melanoma. Peter et al. (9) reported that the expression of ACSM3 was decreased in ulcerative colitis and might be related to butyrate oxidation. However, the effects of ACSM3 on ovarian cancer has not been reported.

Integrin $\beta 1$ (ITG $\beta 1$ ), a member of the heterodimeric transmembrane receptors family, is known as a cellextracellular matrix (ECM) adhesion protein (10). Integrin $\beta 1$ exerts regulatory functions on cellular processes including cell proliferation, migration, and invasion via the signals reacted to ECM during cancer progression (10). Studies indicated that ITG $\beta 1$ (Integrin $\beta 1$ ) had an up-regulation in ovarian cancer tissues and the overexpression of ITG $\beta 1$ (Integrin $\beta 1$ ) was linked with poor prognosis as well as higher clinical stages $(11,12)$. The upregulated expression of ITG $\beta 1$ (Integrin $\beta 1$ ) elevates the abilities of cell migration and invasion in ovarian cancer tumor (11).

In this work, we found that the expression of ACSM3 was down-regulated in ovarian cancer by bioinformatics analysis, and the patients with low expression of ACSM3 showed poor overall survival. We artificially regulated the expression of ACSM3 to evaluate the effects on ovarian cancer progression. The modulation of ACSM 3 markedly influenced cell proliferation, metastasis, and invasion in vitro as well as the growth of ovarian cancer tumors in vivo. Further work showed that these effects are associated with its regulation on the Integrin $\beta 1 / \mathrm{AKT}$ signaling pathway.

\section{MATERIALS AND METHODS}

\section{Cell Culture, Vectors Construction, and Cell Transfection}

OV-90, SK-OV-3, OVCAR-3, and A2780 cells were purchased from Procell Life Science\&Technology Co., Ltd (Wuhan, China).
OV-90 and OVCAR-3 cells were maintained in a specific medium (Procell). SK-OV-3 cells were cultured in McCoy's 5A medium (Procell). A2780 cells were cultured in DEME (Gibco Life Technologies, NY, USA). All the medium was supplemented with $10 \%$ fetal bovine serum (FBS). And cells were incubated in $5 \% \mathrm{CO}_{2}$ at $37^{\circ} \mathrm{C}$.

Lentiviral vectors expressing small hairpin RNA (shRNA) targeting ACSM3 were named Lv-shRNA1-ACSM3 or LvshRNA2-ACSM3. The complementary cDNAs of ACSM3 were synthesized and the lentiviral overexpressed vectors pcDNA3.1 (GenScript, Nanjing, China) of ACSM3 were constructed as LvACSM3. The shRNA sequences were listed as follow. shRNA1ACSM3: CGATGTTAAGATTGTAGATGT. shRNA2-ACSM3: GCTTGTACAGAATGATATAAC. The Lv-shRNA1-ACSM3 or the Lv-shRNA2-ACSM3 were infected into OV-90 cells. The Lv-ACSM3 was infected into A2780 or SK-OV-3 cells. The overexpressed ITG $\beta 1$ (Integrin $\beta 1$ ) vectors were transfected into A2780 cells by Lipofectamine 3000 (Invitrogen, Carlsbad, California, USA) according to the manufacturer's instructions.

\section{Western Blot}

Total protein was extracted from infected cells or tumors, respectively. The protein concentration was measured by BCA Protein Assay Kit (Solarbio, Shanghai, China). $20 \mu \mathrm{L}$ protein samples were resolved by $12 \%$ SDS-PAGE and transferred onto PVDF membranes (Millipore, Billerica, MA, USA). Protein bands were incubated with primary antibodies at $4^{\circ} \mathrm{C}$ overnight after being blocked with $5 \%$ skim milk. Then, membranes were incubated with HRP labeled secondary antibodies (Solarbio; $1: 3000$ ) at $37^{\circ} \mathrm{C}$ for an hour. Protein probes were imaged with an ECL reagent (Solarbio). The primary antibodies were ACSM3 (Proteintech. Wuhan, China; 1:500), Integrin $\beta 1$ (Proteintech; 1:500), E-cadherin (ABclonal, Shanghai, China; 1:1000), cyclin D1 (ABclonal; 1:500), p-AKT (ABclonal; 1:500), AKT (ABclonal; 1:1000), Vimentin (ABclonal; $1: 500)$, c-Myc (ABclonal; 1:500), and GAPDH (Proteintech; 1:10000).

\section{Cell Counting Kit-8 Assay (CCK-8 assay)}

The ability of cell proliferation was detected by CCK- 8 assay. Infected cells $\left(4 \times 10^{3}\right.$ per well) were plated into 96 -well plates and were cultured for $0 \mathrm{~h}, 24 \mathrm{~h}, 48 \mathrm{~h}, 72 \mathrm{~h}$, and $96 \mathrm{~h}$. Then, a $10 \mu \mathrm{L}$ CCK-8 kit (Keygen Biotech, Jiangsu, China) was added to each well and incubated for two hours. The OD value was measured at $450 \mathrm{~nm}$.

\section{Cell Cycle Analysis}

Nuclear DNA content was evaluated by fluorescence-activated cell sorting. Infected cells were deprived of FBS and were rinsed with phosphate buffer saline (PBS) three times. Cells were maintained in pre-cooling $70 \%$ ethanol at $4^{\circ} \mathrm{C}$ for $2 \mathrm{~h}$ and rinsed with PBS. After being resuspended, cells were incubated with propidium iodide $(50 \mu \mathrm{L} / \mathrm{mL}$; Beyotime, Shanghai, China) and RNAase $\left(0.1 \mathrm{mg} / \mathrm{mL}\right.$; Beyotime, Shanghai, China) at $37^{\circ} \mathrm{C}$ 
for half an hour protecting from the light. The nuclear DNA content was measured by performing a NovoCyte flow cytometer (ACEA Biosciences, Hangzhou, China).

\section{Wound Healing Assay}

Equal numbers of OV-90, A2780, and SK-OV-3 cells were plated into 6-well plates after being infected for $24 \mathrm{~h}$, respectively. A wound was achieved by $200 \mu \mathrm{L}$ pipette tips in each well after cells were cultured for $24 \mathrm{~h}$. Cells were rinsed by the medium without serum. The distance between cells was photographed at $0 \mathrm{~h}$ or $24 \mathrm{~h}$, respectively.

\section{Transwell Assay}

After being infected for $24 \mathrm{~h}$, cells were collected and applied to top chambers of transwell inserts (Corning Incorporated, NY, USA) with a non-serum medium. $900 \mu \mathrm{L}$ medium with $10 \%$ FBS was added into lower chambers and the systems were maintained for $24 \mathrm{~h}$. Cells were further stained with $0.5 \%$ crystal violet (Amresco, Shanghai, China). The number of migrated cells in lower chambers was quantified and revealing the invasion ability of cells.

\section{Tumor Xenograft Model}

Six-week-old BALB/c nude mice (Beijing HFK Bioscience, Beijing, China) were cared and manipulated according to the agreement approved by Shengjing Hospital of China Medical University. Infected cells (OV-90 or A2780 cells) in the exponential phase were subcutaneously injected into the armpit. After a week, the tumor diameter was measured every three days and calculated for the tumor volume. Mice were sacrificed on the $23^{\text {rd }}$ after being injected. And the tumors were further used for immunohistochemistry staining.

\section{Immunohistochemistry}

Paraffin-embedded sections of tumors were dewaxed in xylene and then rehydrated in gradient alcohol. Then, sections were further maintained in 3\% hydrogen peroxide for $15 \mathrm{~min}$ to quench the activity of endogenous peroxidase. Sections were incubated with goat serum (Solarbio) for a quarter of an hour at room temperature and then incubated with primary antibodies at $4^{\circ} \mathrm{C}$ overnight. After rinsed by PBS for three times, these sections were incubated with HRP labeled IgG antibodies (Thermo Scientific, Pittsburgh, PA, USA; $1: 500$ ) at $37^{\circ} \mathrm{C}$ for an hour. Followed by DAB color reaction (Solarbio), sections were stained with hematoxylin (Solarbio) and imaged by microscope (Olympus, Tokyo, Japan). The primary antibodies were ACSM3 (Proteintech; 1:50) and Integrin $\beta 1$ (Proteintech; 1:50).

\section{Statistical Analysis}

All statistics were analyzed by using GraphPad Prism 8.0 (GraphPad Software, San Diego, CA, USA). Data were presented as means and standard deviation from at least triple independent experiments. One-way ANOVA was used to evaluate statistical differences in multiple groups except for the results of CCK-8 assay and tumor volume (two-way ANOVA). $\mathrm{p}<0.05$ was considered statistically significant.

\section{RESULTS}

\section{The Expression of ACSM3 and Its Co-relation of the Overall Survival of Ovarian Cancer}

We used Gene Expression Profiling Interactive Analysis (GEPIA; http://gepia.cancer-pku.cn/) to assess the relationship between ACSM3 expression level and overall survival in ovarian cancers by using The Cancer Genome Atlas database. The results showed that ACSM3 was markedly low expressed in ovarian cancer tissues than normal tissues (Figure 1A) Survival analysis revealed that the low expression level of ACSM3 was related with poorer overall survival than high mRNA expression of ACSM3 in 424 ovarian cancer patients, suggesting the prognostic significance of ACSM3. (Figure 1B). We determined the protein expression of ACSM3 in ovarian epithelial cells and ovarian cancer cells: OV-90, SK-OV-3, OVCAR-3, and A2780 cells. ACSM3 was highly expressed in OV90 cells and was low expressed in A2780 and SK-OV-3 cells (Figure 1C). Then, we artificially regulated the expression of ACSM 3 in OV90, A2780, and SK-OV-3 cells. Western bolt demonstrated that the knockdown of ACSM3 in OV-90 cells and the overexpression of ACSM3 in A2780 or SK-OV-3 cells were successful (Figures 1D, E). It could be seen that the expression of ACSM3 was downregulated in OV-90 cells with a highly expressed baseline of ACSM3 and was upregulated in SK-OV-3 and A2780 cells with a reduced baseline expression of ACSM3 (Figures 1D, E).

\section{Effects of ACSM3 on the Proliferation of Ovarian Cancer Cells}

CCK-8 assay and clone formation assay were performed to measure the ability of cell proliferation in ovarian cancer cells. The results of the CCK- 8 assay showed that the knockdown of ACSM3 promoted the growth of OV-90 cells significantly, and the overexpression of ACSM3 markedly inhibited the ability of cell proliferation in A2780 or SK-OV-3 cells (Figures 2A-C). Clone formation assay demonstrated that the clone forming ability of OV-90 cells was elevated following ACSM3 knockdown, while the clone forming ability of A2780 or SK$\mathrm{OV}-3$ cells was decreased by the up-regulation of ACSM3 (Figures 2D-F). To investigate the effects of ACSM3 on cell cycle arrest, cellular DNA content was detected by flow cytometry in OV-90, A2780, or SK-OV-3 cells. The results showed that the cells in the G1 phase were reduced accompanied by the elevation of cells in the $S$ phase in OV-90 cells induced by the interference of ACSM3 (Figure 2G). And the cells in the G1 phase were enhanced with an attenuation of cells in the S phase and G2 phase in A2780 or SK-OV-3 cells infected with overexpressed ACSM3 (Figures 2H, I). These results indicated ACSM3 repressed the proliferation ability of ovarian cancer cells.

\section{Effects of ACSM3 on Migration and Invasion of Ovarian Cancer Cells}

The ability of cell migration and invasion was evaluated by the wound healing assay and the transwell assay. The results of the wound healing assay verified that cell migration ability was 
A

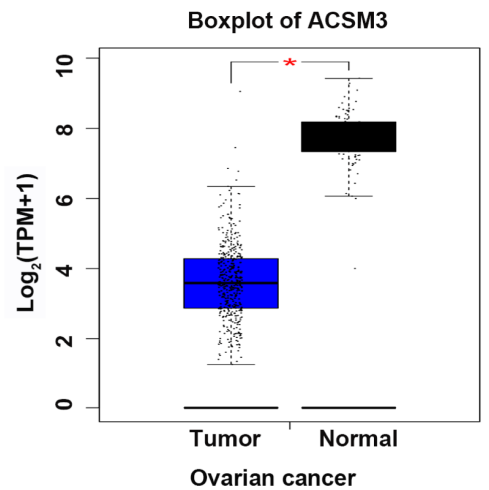

B

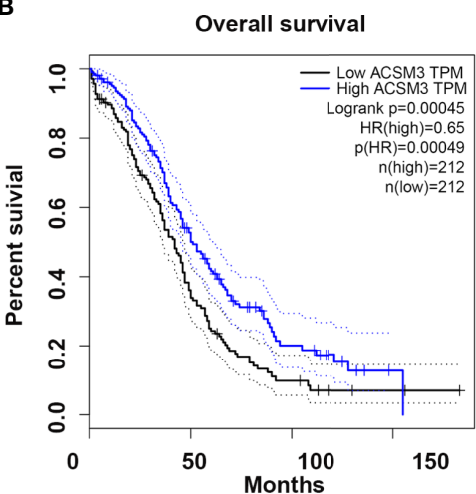

(num $(T)=426 ; \operatorname{num}(N)=88$ )
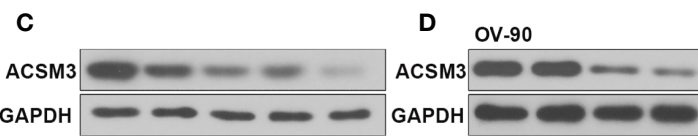

E $\quad$ A2780
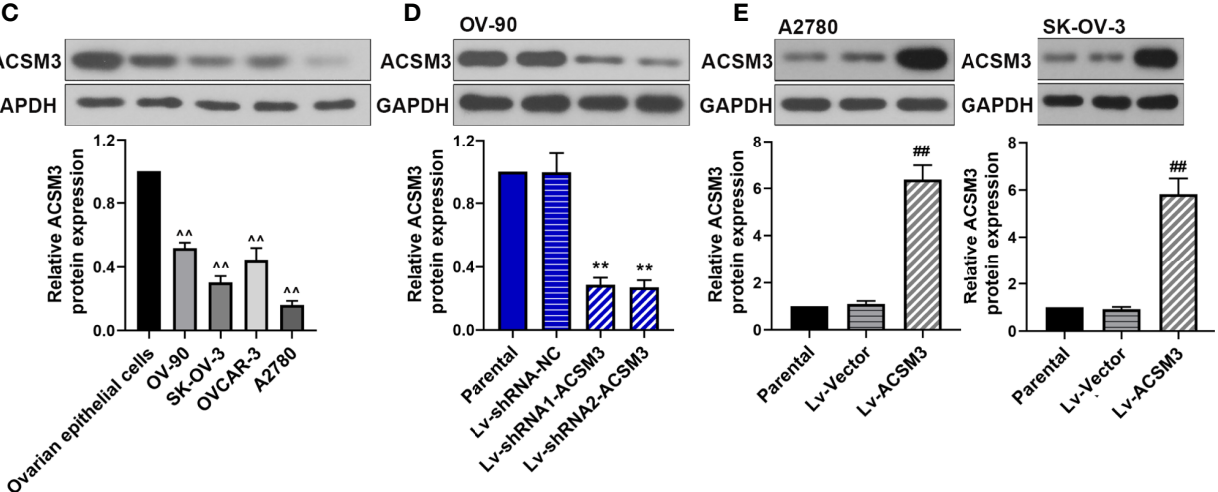

FIGURE 1 | The expression of ACSM3 and its co-relation of the overall survival of ovarian cancer. (A) Boxplot of ACSM3 in ovarian cancer. TPM, transcripts per kilobase of exon model per million mapped reads. (B) Overall survival time between patients with high and low ACSM3 expression. Dotted lines indicated the 95\% confidence interval. HR, hazard ratio. (C) Relative protein expression of ACSM3 in ovarian epithelial cells and ovarian cancer cells: OV-90, SK-OV-3, OVCAR-3, and A2780 cells. (D) Relative protein expression of ACSM3 in LV-shRNA-ACSM3 infected OV-90 cells (E) Relative protein expression of ACSM3 in LV-ACSM3 infected A2780 or SK-OV-3 cells. Measurement data were expressed as mean \pm SD of three independent experiments. $\wedge \wedge p<0.01$ versus ovarian epithelial cells; ${ }^{\star} p<0.05$, ${ }^{* \star} p<0.01$ versus Lv-shRNA-NC; ${ }^{\# \#} p<0.01$ versus Lv-Vector.

enhanced in OV-90 cells induced by the interference of ACSM3, while cell migration ability was weakened in A2780 or SK-OV-3 cells infected with Lv-ACSM3 (Figures 3A-C). The results of the transwell assay showed that infected with ACSM3 shRNAs increased the invasion ability of OV-90 cells, while the overexpression of ACSM3 decreased the invasion ability of A2780 cells or SK-OV-3 cells (Figures 3D-F). These data suggested that ACSM3 decreased the migration and invasion abilities of ovarian cancer cells.

\section{Effects of ACSM3 on the Integrin $\beta 1 / \mathrm{AKT}$ Signaling Pathway in Ovarian Cancer}

To further explore the mechanism of the effects caused by $A C S M 3$, the expression of key proteins (Integrin $\beta 1, \mathrm{p}-\mathrm{AKT}$, and $\mathrm{AKT}$ ) in the Integrin $\beta 1 / \mathrm{AKT}$ signaling pathway was evaluated. Western blot demonstrated that the knockdown of ACSM3 promoted the expression of Integrin $\beta 1$ and $\mathrm{p}-\mathrm{AKT}$ in OV-90 cells, leading to the up-regulation of cyclin D1, c-Myc, Vimentin, and the down-regulation of E-cadherin (Figure 4A). The overexpression of ACSM3 attenuated the expression of Integrin $\beta 1$ and $\mathrm{p}-\mathrm{AKT}$ in A2780 cells, causing the low expression of cyclin D1, c-Myc, Vimentin, and the high expression of E-cadherin (Figure 4B). Our results suggested the possibility that ACSM3 attenuated the expression of cell proliferation or metastasis-related proteins by the inhibition of the Integrin $\beta 1 / \mathrm{AKT}$ signaling pathway in ovarian cancer.

\section{Effects of ITG $\beta 1$ (Integrin $\beta 1$ ) on ACSM3 Up-regulated Ovarian Cancer Cells}

To further investigate the effects of Integrin $\beta 1$ on ACSM3 upregulated ovarian cancer cells, ITG $\beta 1$ (Integrin $\beta 1$ ) overexpressed plasmids were constructed and transfected to A2780 cells. Western blot proved that the construction and transfection of overexpressed plasmids were successful in A2780 cells (Figure 5A). The results of the CCK-8 assay revealed that the up-regulated expression of ACSM3 reduced the growth of A2780 cells. Conversely, the overexpression of ITG $\beta 1$ (Integrin $\beta 1$ ) reversed the alleviation of cell proliferation ability (Figure 5B). ACSM3 overexpression-induced suppression of cell migration activity was abolished by the overexpression of ITG $\beta 1$ (Integrin $\beta 1$ ) (Figures 5C, D). Moreover, the overexpression of ITG $\beta 1$ (Integrin $\beta 1$ ) reversed the alleviated 
A

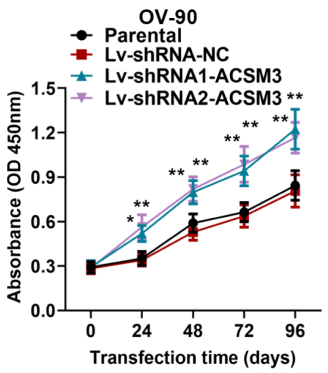

D

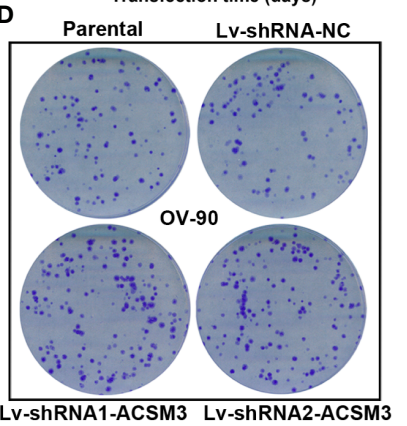

OV-90

- 1 Parental

2 Lv-shRNA-NC

ש 3 Lv-ShRNA1-ACSM3
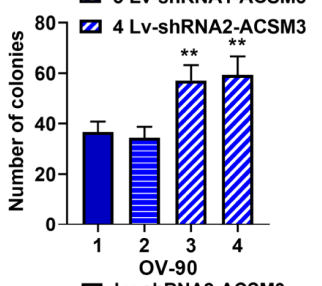

G

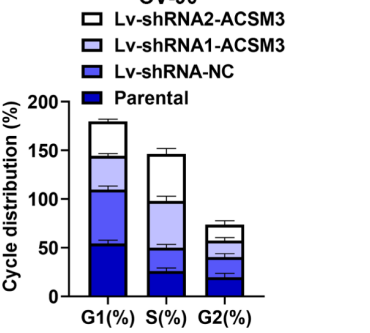

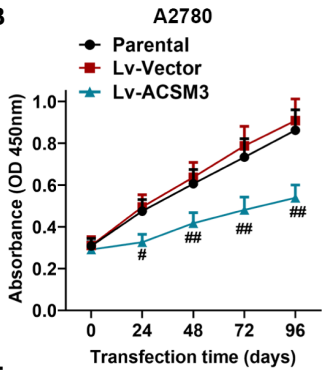

E Parental

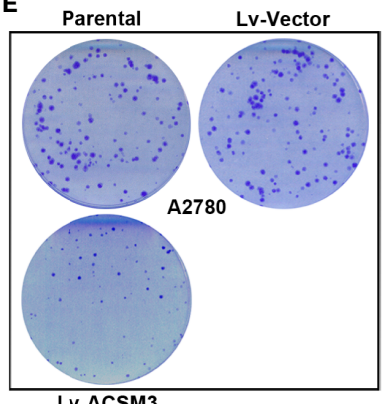

Lv-ACSM3

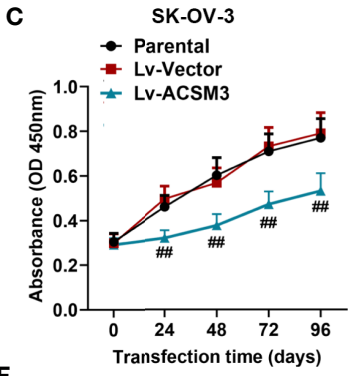

F

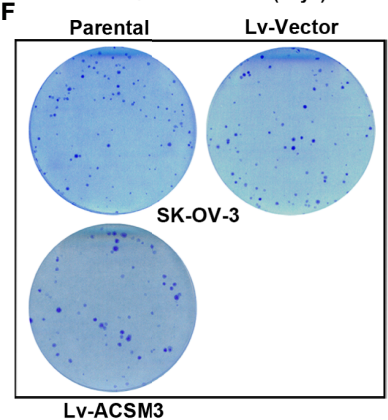

Lv-ACSM3
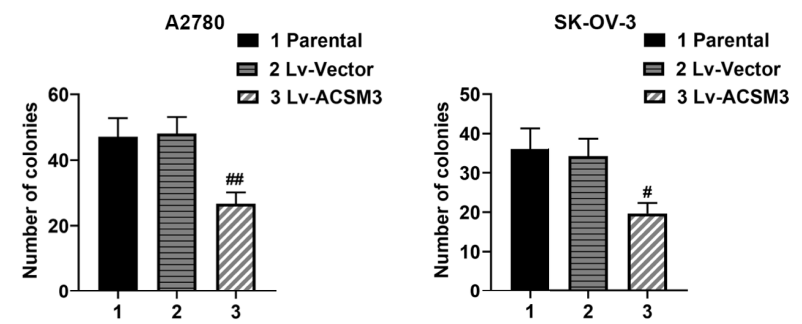

H
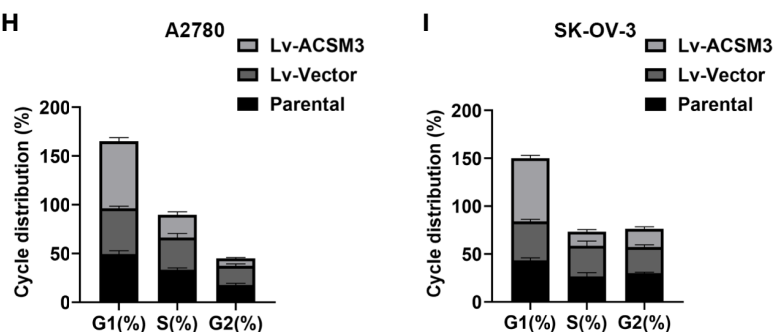

FIGURE 2 | Effects of ACSM3 on the proliferation of ovarian cancer cells. OV-90 cells were infected with ACSM3 shRNAs. A2780 or SK-OV-3 cells were infected with overexpressed ACSM3 vectors. (A-C) OV-90, A2780, or SK-OV-3 cells were subjected to CCK-8 assay. (D-F) The number of colonies. (G-I) Cell cycle arrest was detected by the flow cytometer. Measurement data were expressed as mean \pm SD of three independent experiments. ${ }^{*} \mathrm{p}<0.05$, ${ }^{\star *} \mathrm{p}<0.01$ versus $\mathrm{LV}$-shRNA$\mathrm{NC} ;{ }^{\#} \mathrm{P}<0.05,{ }^{\# \#} \mathrm{P}<0.01$ versus Lv-Vector.

cell invasion ability in A2780 cells infected with Lv-ACSM3 (Figures 5E, F). Then, we evaluated the effects of overexpressed ITG $\beta 1$ (Integrin $\beta 1$ ) on the Integrin $\beta 1 / \mathrm{AKT}$ signaling pathway. ACSM3 overexpression-induced low expression of p-AKT was reversed by the overexpression of ITG $\beta 1$ (Integrin $\beta 1$ ), causing the up-regulation of the low expression of cyclin D1, c-Myc, Vimentin and the down-regulation of high expression of Ecadherin (Figure 5G). These data confirmed that the overexpressed ITG $\beta 1$ (Integrin $\beta 1$ ) could reverse the malignant process caused by the up-regulated ACSM3 in ovarian cancer.

\section{Effects of ACSM3 on the Growth of Ovarian Cancer Tumor In Vivo}

To confirm the effects of ACSM3 on the growth of ovarian cancer tumor in vivo, a xenograft tumor model was built with OV-90 cells infected with Lv-shRNA1-ACSM3 or A2780 cells infected with LvACSM3. Compared to Lv-Vector, the overexpression of ACSM3 significantly ameliorated the average tumor volume (Figures 6A, B). However, compared to Lv-shRNA-NC, ACSM3 interference markedly increased the average tumor volume (Figures 6A, B). Then, we verified the expression of ACSM3 and Integrin $\beta 1$ by performing immunohistochemistry. The results indicated that the 


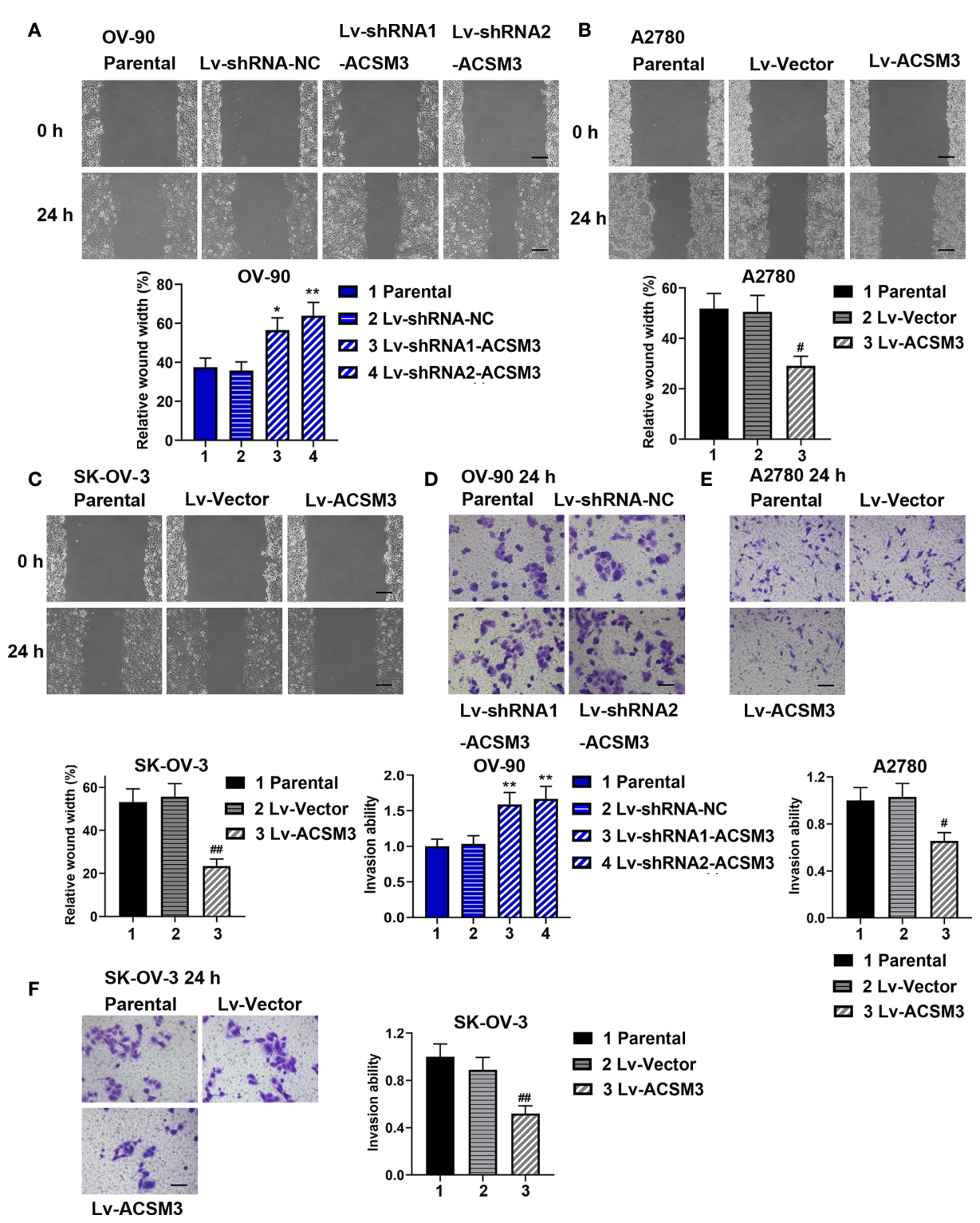

FIGURE 3 | Effects of ACSM3 on migration and invasion of ovarian cancer cells. OV-90 cells were infected with LV-shRNA-ACSM3. A2780 or SK-OV-3 cells were infected with LV-ACSM3. (A-C) Cell migration ability was measured by wound healing assay. Scale bar represented $200 \mu \mathrm{m}$ (D-F) The ability of cell invasion was detected by transwell assay. Scale bar represented $100 \mu \mathrm{m}$. Measurement data were expressed as mean $\pm \mathrm{SD}$ of three independent experiments. ${ }^{*} p<0.05$, ${ }^{* *} \mathrm{P}<0.01$ versus LV-shRNA-NC; ${ }^{\#} \mathrm{P}<0.05,{ }^{\# \#} \mathrm{P}<0.01$ versus $\mathrm{Lv}$-Vector.

expression of ACSM3 was reduced, while the expression of Integrin $\beta 1$ was enhanced in tumors derived from the Lv-shRNA1-ACSM3infected OV-90 cells model (Figure 6C). The expression of ACSM3 was increased, while the expression of Integrin $\beta 1$ was alleviated in tumors derived from the Lv-Vectors-infected A2780 cells model (Figure 6C). We further proved the association between ACSM3 and Integrin $\beta 1 / \mathrm{AKT}$ signaling pathway. The protein expression of p-AKT was elevated in tumors derived from the Lv-shRNA1ACSM3-infected OV-90 cells model, causing the up-regulation of $\mathrm{c}-\mathrm{Myc}$ and Vimentin (Figure 6D). The protein expression of $\mathrm{p}$ AKT was weakened in tumors derived from the Lv-Vectorsinfected A2780 cells model, leading to the attenuated expression of c-Myc and Vimentin (Figure 6E). The results suggested that ACSM3 inhibited the growth of ovarian cancer tumors in vivo via the abrogation of the Integrin $\beta 1 / \mathrm{AKT}$ signaling axis.

\section{DISCUSSION}

In this study, we found that the expression of ACSM3 was downregulated in ovarian cancer by bioinformatics analysis, and the patients with low expression of ACSM3 showed poor overall survival. We artificially regulated the expression of ACSM3 to evaluate the effects on ovarian cancer progression. Our data 


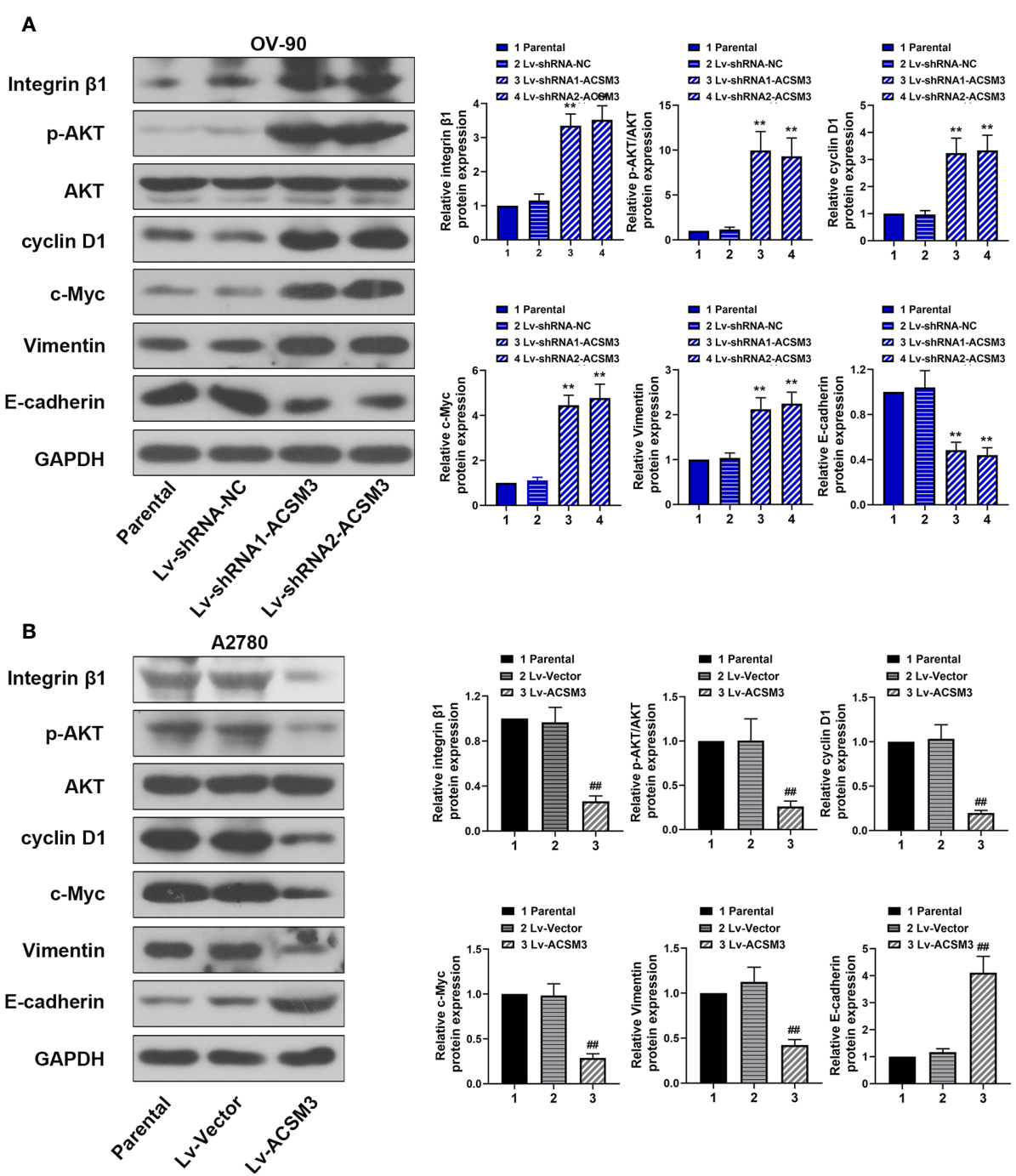

FIGURE 4 | Effects of ACSM3 on the Integrin $\beta 1 / A K T$ signaling pathway in ovarian cancer. (A) The protein expression of Integrin $\beta 1$, p-AKT, AKT, cyclin D1, C-Myc, Vimentin, and E-cadherin in OV-90 cells following ACSM3 knockdown. (B) The protein expression of Integrin $\beta 1$, p-AKT, AKT, cyclin D1, c-Myc, Vimentin, and Ecadherin in A2780 cells following ACSM3 overexpression. Measurement data were expressed as mean $\pm \mathrm{SD}$ of three independent experiments. ${ }^{\star *} \mathrm{P}<0.01$ versus Lv-shRNA-NC; \#\# $\mathrm{P}<0.01$ versus Lv-Vector.

revealed that the overexpression of ACSM3 inhibited cell proliferation, migration, and invasion in ovarian cancer mediated by the suppression of the Integrin $\beta 1 /$ AKT signaling pathway in vitro. While the knockdown of ACSM 3 enhanced the ovarian cancer progression via the activity of the Integrin $\beta 1 / \mathrm{AKT}$ signaling axis. Moreover, ACSM3 overexpression-induced suppression of cell migration and invasion activities were abolished by the overexpression of ITG $\beta 1$ (Integrin $\beta 1$ ) in vitro. Then, we found that the overexpression of ACSM3 ameliorated the growth of ovarian cancer xenograft tumors in vivo. Conversely, ACSM3 interference facilitated the growth of tumors in ovarian cancer.

Studies have shown that the dysregulated expression of acylCoA synthetases family subunits exists in many diseases. Acyl-CoA synthetase long-chain family member 4 (ACSL4) is overexpressed in breast and prostate cancer (13). Very long-chain Acyl-CoA synthetase homology 3 (ACSVL3) is seen as a biomarker for targeted therapy in lung cancer (14). Gopal et al (15) found that ACSM3 was low expressed in hepatocellular carcinoma and activated the WNT/AKT signaling axis. ACSM3 was reported to have a down-regulated expression in cutaneous melanoma and Duchenne muscular dystrophy $(8,16)$. However, there have been no studies on the expression of ACSM3 in ovarian cancer. In this work, we found that the expression of ACSM3 was down-regulated in ovarian cancer tissues than normal tissues. Furthermore, ACSM3 has been reported to co-related with poor prognosis in malignant melanoma and liver cancer $(7,8)$. The survival analysis from the public database showed that the low level of ACSM3 is significantly correlated with the poor overall survival of ovarian cancer, thus, we 


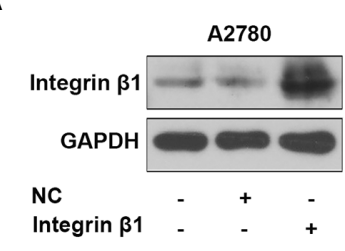

C

$\mathrm{Oh}$

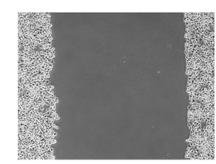

\section{Ne}
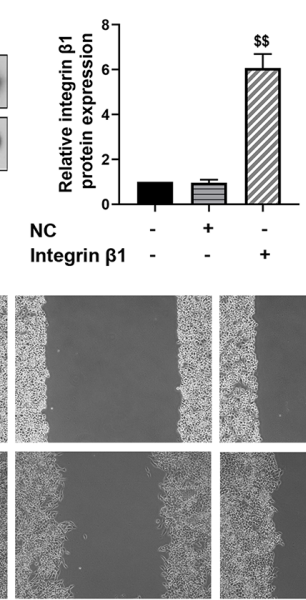

$+$ $\begin{array}{ll}+ & + \\ + & +\end{array}$

E Lv-ACSM3
NC
Integrin $\beta 1$

$24 \mathrm{~h}$

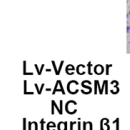

$N C$
Integrin $\beta 1$
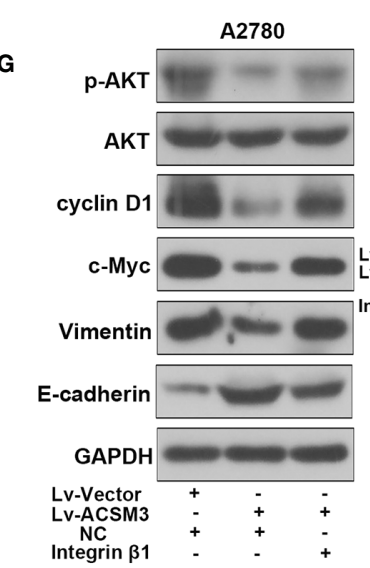
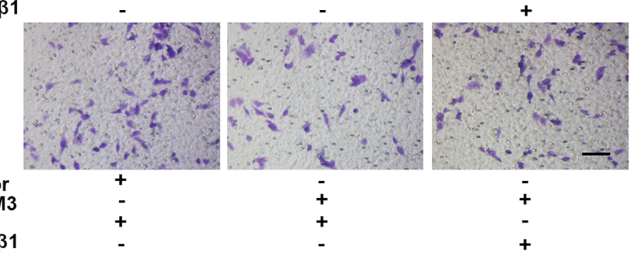

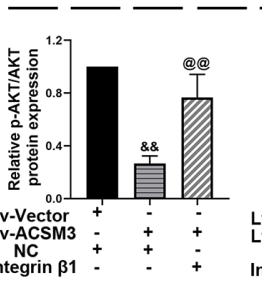

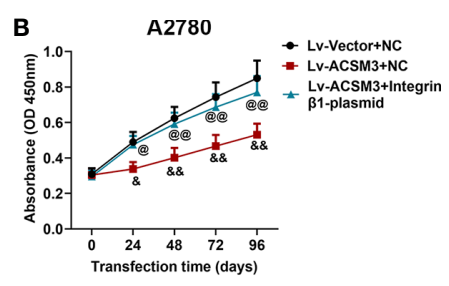
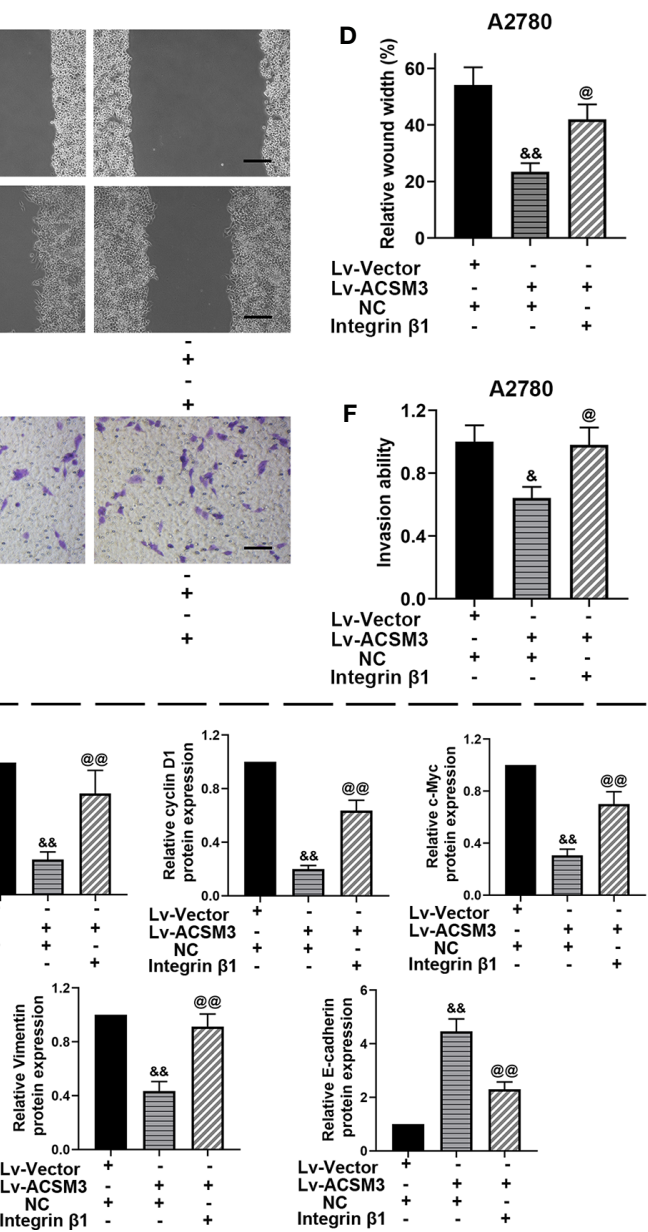

FIGURE 5 | Effects of overexpressed ITG $\beta 1$ (Integrin $\beta 1$ ) on ACSM3 up-regulated ovarian cancer cells. (A) The protein expression of Integrin $\beta 1$. (B) ACSM3 upregulated A2780 cells were transfected by ITG $\beta 1$ (Integrin $\beta 1$ ) overexpression vector and were subjected to CCK-8 assay. (C, D) Relative wound width (Scale bar represented $200 \mu \mathrm{m})$. (E, F) Invasion ability. Scale bar represented $100 \mu \mathrm{m}$. (G) Relative protein expression of p-AKT, AKT, cyclin D1, c-Myc, Vimentin, and Ecadherin. Measurement data were expressed as mean \pm SD of three independent experiments. ${ }^{\$ \$} p<0.01$ versus NC; ${ }^{\&} p<0.05,{ }^{8 \&} p<0.01$ versus $L v$-Vector+NC; ${ }^{\circledR} \mathrm{p}<0.05,{ }^{\circledR} \mathrm{p}<0.01$ versus Lv-ACSM+NC.

have reasons to speculate that the ACSM3 is related to the clinic malignancy grade of ovarian cancer. We will attach much importance to this part of the research, and further investigation will be conducted.

In high-grade serous ovarian carcinomas, tumors proliferate rapidly and disseminate early, as well as have an aggressive duration of disease (17). Thus, the inhibition of metastasis is the point for alleviating the disease course of ovarian cancer and for further treatment. Ruan et al (7) reported that the loss of ACSM3 elevated the metastasis in liver cancer. Zhu et al (8) have proven that the
ACSM3 overexpression decreased the proliferation, invasion, and colony formation in malignant melanoma in vitro. In this research, we artificially regulated the expression of ACSM3 to determine the effects it on the progression of ovarian cancer in vitro or in vivo. The results revealed that the overexpression of ACSM3 significantly decreased the abilities of ovarian cell proliferation, migration, and invasion in vitro, and weakened the growth of ovarian cancer tumors in vivo.

Furthermore, we determined the mechanism that ACSM3 acted as an antioncogene in ovarian cancer. The up-regulated 


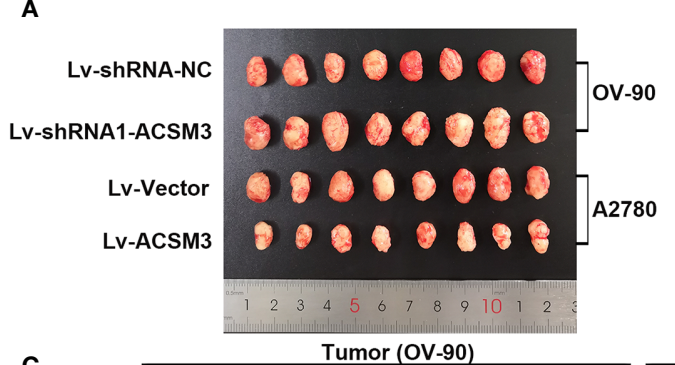

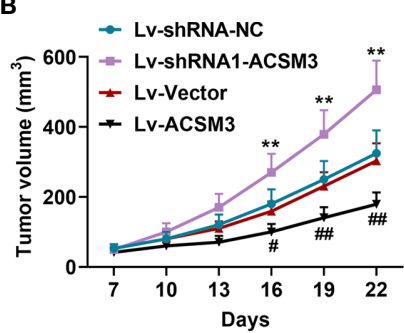

C

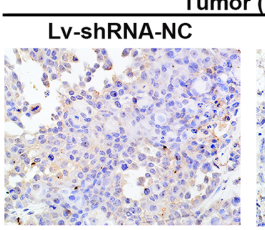

Lv-shRNA1-ACSM3

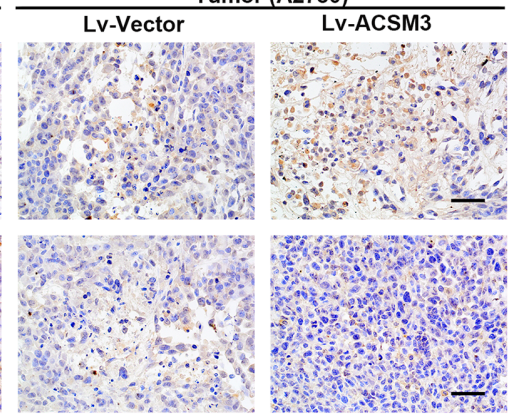

D
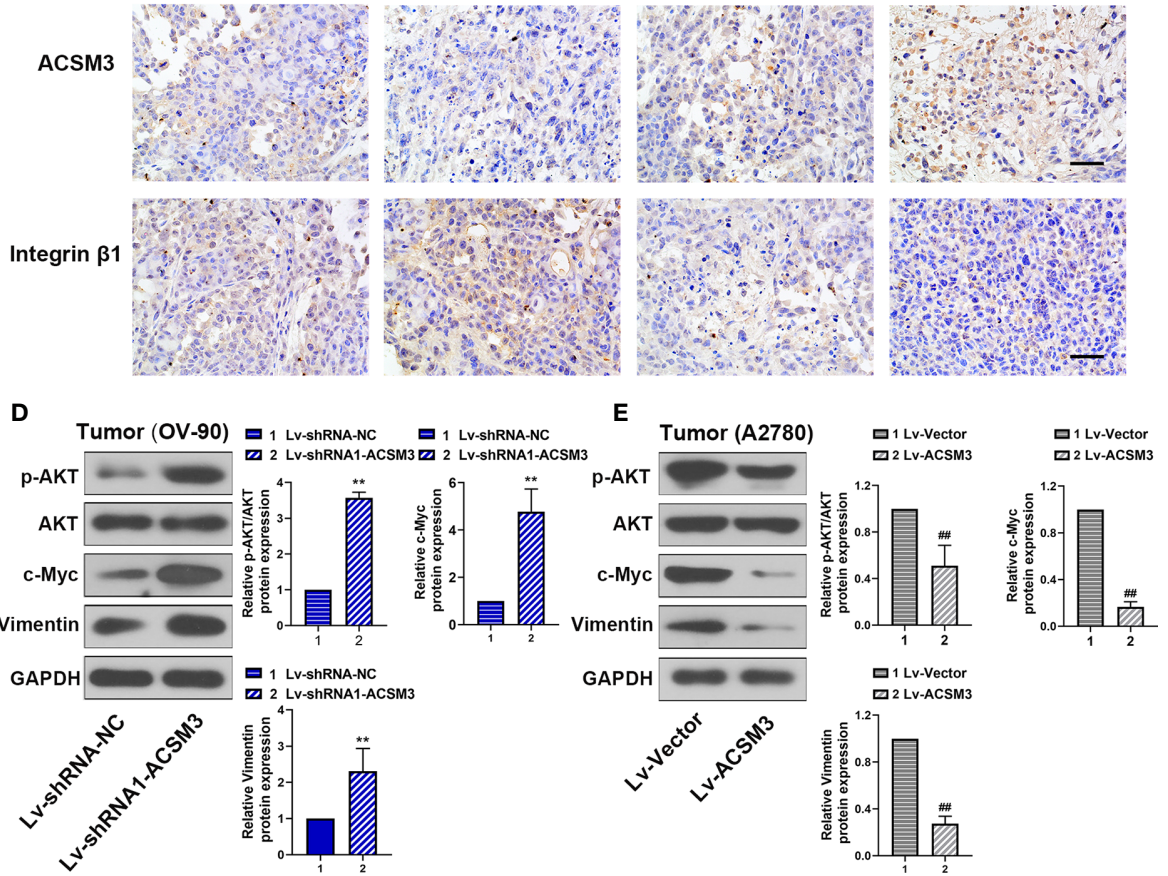

FIGURE 6 | Effects of ACSM3 on the growth of ovarian cancer tumor in vivo. A xenograft tumor model was built with OV-90 cells infected with LV-shRNA1-ACSM3 or A2780 cells infected with LV-ACSM3. (A, B) The volume of xenograft tumors. (C) The expression of ACSM3 and Integrin $\beta 1$ was measured by immunohistochemistry. Scale bar represented $50 \mu \mathrm{m}$. (D, E) Relative protein expression of $\mathrm{p}-\mathrm{AKT}, \mathrm{AKT}, \mathrm{c}-\mathrm{Myc}$, Vimentin, and E-cadherin. Measurement data were expressed as mean $\pm S D$ of six independent experiments. ${ }^{* *} p<0.01$ versus $L v-s h R N A-N C ;{ }^{\#} p<0.05,{ }^{\# \#} p<0.01$ versus Lv-Vector.

expression of ITG $\beta 1$ (Integrin $\beta 1$ ) has been proven to be linked with ovarian cancer progressions such as cell proliferation, metastasis, and invasion $(18,19)$. AKT is known as a vital part of the cell cycle, cell survival, and apoptosis, and is positively associated with Integrin $\beta 1$ (20). Studies revealed that the high expression of Ecadherin and the low expression of Vimentin cause epithelial differentiation, which is essential for tumor metastasis and invasion (21). The gain of cyclin D1 and c-Myc are associated with cell proliferation and malignant transformation (22). Zhang et al (23) reported that the loss of the Integrin $\beta 1 / F A K / A K T$ signaling axis caused the down-regulation of cyclin D1 and collagen in hepatic fibrosis rats. Riggio et al. (24) pointed out that the AKT1 enhanced cell proliferation via the up-regulated cyclin D1 and S6, and then suppressed the cell migration and invasion via the downregulated Integrin $\beta 1$ and FAK. Bartolomé et al. (25) demonstrated that integrin signal specifically activated AKT and JNK mediated by vascular-endothelial (VE)-cadherin, which was associated with metastatic dissemination in melanoma. Here, we found that the overexpressed ACSM3 blocked the expression of Integrin $\beta 1$ and $\mathrm{p}$ AKT, leading to the down-regulation of cyclin D1, c-Myc, Vimentin and the up-regulation of E-cadherin in ovarian cancer cells. These results indicated that up-regulated ACSM3 inhibited the cell proliferation, migration, and invasion via the Integrin $\beta 1 / \mathrm{AKT}$ signaling pathway in ovarian cancer. Moreover, the ACSM3 overexpression-induced suppression of cell migration and invasion activities were abolished by the overexpression of ITG $\beta 1$ (Integrin $\beta 1$ ).

\section{CONCLUSION}

ACSM3 was low expressed in ovarian cancer tissues. The overexpression of ACSM3 inhibits the malignant phenotypes 
via the Integrin $\beta 1 / \mathrm{AKT}$ signaling axis in ovarian cancer. Furthermore, ACSM3 could be a potential therapeutic target in ovarian cancer.

\section{DATA AVAILABILITY STATEMENT}

The raw data supporting the conclusions of this article will be made available by the authors, without undue reservation.

\section{ETHICS STATEMENT}

The animal study was reviewed and approved by the Ethics Committee of China Medical University.

\section{REFERENCES}

1. Miller KD, Nogueira L, Mariotto AB, Rowland JH, Yabroff KR, Alfano CM, et al. Cancer treatment and survivorship statistics, 2019. CA Cancer J Clin (2019) 69(5):363-85. doi: 10.3322/caac.21565

2. Matulonis UA, Sood AK, Fallowfield L, Howitt BE, Sehouli J, Karlan BY. Ovarian cancer. Nat Rev Dis Primers (2016) 2:16061. doi: 10.1038/ nrdp. 2016.61

3. Cheng L, Wu S, Zhang K, Ya Q, Xu T. A comprehensive overview of exosomes in ovarian cancer: emerging biomarkers and therapeutic strategies. J Ovarian Res (2017) 10(1):73. doi: 10.1186/s13048-017-0368-6

4. Chien J, Poole EM. Ovarian Cancer Prevention, Screening, and Early Detection: Report From the 11th Biennial Ovarian Cancer Research Symposium. Int J Gynecol Cancer (2017) 27(9S Suppl 5):S20-S2. doi: 10.1097/IGC.0000000000001118

5. Weidle UH, Birzele F, Kollmorgen G, Rueger R. Mechanisms and Targets Involved in Dissemination of Ovarian Cancer. Cancer Genomics Proteomics (2016) 13(6):407-23. doi: 10.21873/cgp.20004

6. Lengyel E. Ovarian cancer development and metastasis. Am J Pathol (2010) 177(3):1053-64. doi: 10.2353/ajpath.2010.100105

7. Ruan H-Y, Yang C, Tao X-M, He J, Wang T, Wang H, et al. Downregulation of ACSM3 promotes metastasis and predicts poor prognosis in hepatocellular carcinoma. Am J Cancer Res (2017) 7(3):543-53.

8. Zhu Z, Wang D, Shen Y. Loss of ACSM3 confers worsened prognosis and immune exclusion to cutaneous melanoma. J Cancer (2020) 11(22):6582-90. doi: $10.7150 /$ jca. 48354

9. De Preter V, Arijs I, Windey K, Vanhove W, Vermeire S, Schuit F, et al. Impaired butyrate oxidation in ulcerative colitis is due to decreased butyrate uptake and a defect in the oxidation pathway. Inflammation Bowel Dis (2012) 18(6):1127-36. doi: 10.1002/ibd.21894

10. Desgrosellier JS, Cheresh DA. Integrins in cancer: biological implications and therapeutic opportunities. Nat Rev Cancer (2010) 10(1):9-22. doi: 10.1038/ nrc2748

11. Lau M-T, So W-K, Leung PCK. Integrin $\beta 1$ mediates epithelial growth factorinduced invasion in human ovarian cancer cells. Cancer Lett (2012) 320 (2):198-204. doi: 10.1016/j.canlet.2012.02.028

12. Zhang $L$, Zou W. Inhibition of integrin $\beta 1$ decreases the malignancy of ovarian cancer cells and potentiates anticancer therapy via the FAK/STAT1 signaling pathway. Mol Med Rep (2015) 12(6):7869-76. doi: 10.3892/mmr.2015.4443

13. Monaco ME, Creighton CJ, Lee P, Zou X, Topham MK, Stafforini DM. Expression of Long-chain Fatty Acyl-CoA Synthetase 4 in Breast and Prostate Cancers Is Associated with Sex Steroid Hormone Receptor Negativity. Transl Oncol (2010) 3(2):91-8. doi: 10.1593/tlo.09202

14. Pei Z, Fraisl P, Shi X, Gabrielson E, Forss-Petter S, Berger J, et al. Very longchain acyl-CoA synthetase 3: overexpression and growth dependence in lung cancer. PloS One (2013) 8(7):e69392. doi: 10.1371/journal.pone.0069392

15. Gopal R, Selvarasu K, Pandian PP, Ganesan K. Integrative transcriptome analysis of liver cancer profiles identifies upstream regulators and clinical

\section{AUTHOR CONTRIBUTIONS}

LY performed the experiments and wrote the manuscript. $\mathrm{ZH}$ and WL performed the statistical analysis. NL wrote sections of the manuscript. SG designed the experiment and made revision of the manuscript. All authors contributed to the article and approved the submitted version.

\section{FUNDING}

This study was supported by grants from the 345 Talent Project, Shengjing Hospital of China Medical University and Young Backbone Teachers Projects of China Medical University QGZD2018063, 345 Talent Project.

significance of ACSM3 gene expression. Cell Oncol (Dordr) (2017) 40(3):21933. doi: 10.1007/s13402-017-0321-0

16. Dowling P, Zweyer M, Raucamp M, Henry M, Meleady P, Swandulla D, et al. Proteomic and cell biological profiling of the renal phenotype of the $\mathrm{mdx}-4 \mathrm{cv}$ mouse model of Duchenne muscular dystrophy. Eur J Cell Biol (2020) 99 (1):151059. doi: 10.1016/j.ejcb.2019.151059

17. Lisio M-A, Fu L, Goyeneche A, Gao Z-H, Telleria C. High-Grade Serous Ovarian Cancer: Basic Sciences, Clinical and Therapeutic Standpoints. Int Mol Sci (2019) 20(4):952. doi: 10.3390/ijms20040952

18. Zeng B, Zhou M, Wu H, Xiong Z. SPP1 promotes ovarian cancer progression via Integrin $\beta 1 / F A K / A K T$ signaling pathway. Onco Targets Ther (2018) 11:1333-43. doi: 10.2147/OTT.S154215

19. Yang Z, Zhou X, Liu Y, Gong C, Wei X, Zhang T, et al. Activation of integrin $\beta 1$ mediates the increased malignant potential of ovarian cancer cells exerted by inflammatory cytokines. Anticancer Agents Med Chem (2014) 14(7):95562. doi: $10.2174 / 1871520614666140613123108$

20. Revathidevi S, Munirajan AK. Akt in cancer: Mediator and more. Semin Cancer Biol (2019) 59:80-91. doi: 10.1016/j.semcancer.2019.06.002

21. Paolillo M, Schinelli S. Extracellular Matrix Alterations in Metastatic Processes. Int J Mol Sci (2019) 20(19):4947. doi: 10.3390/ijms20194947

22. Karkhanis V, Alinari L, Ozer HG, Chung J, Zhang X, Sif S, et al. Protein arginine methyltransferase 5 represses tumor suppressor miRNAs that down-regulate CYCLIN D1 and c-MYC expression in aggressive B-cell lymphoma. J Biol Chem (2020) 295(5):1165-80. doi: 10.1074/ jbc.RA119.008742

23. Zhang K, Jiang M-n, Zhang C-h, Li C, Jia Y-j. Effects of Ganfukang on expression of connective tissue growth factor and focal adhesion kinase/ protein kinase B signal pathway in hepatic fibrosis rats. Chin J Integr Med (2014) 20(6):438-44. doi: 10.1007/s11655-013-1597-1

24. Riggio M, Perrone MC, Polo ML, Rodriguez MJ, May M, Abba M, et al. AKT1 and AKT2 isoforms play distinct roles during breast cancer progression through the regulation of specific downstream proteins. Sci Rep (2017) 7:44244. doi: 10.1038/srep44244

25. Bartolomé RA, Torres S, Isern de Val S, Escudero-Paniagua B, Calviño E, Teixidó J, et al. VE-cadherin RGD motifs promote metastasis and constitute a potential therapeutic target in melanoma and breast cancers. Oncotarget (2017) 8(1):215-27. doi: 10.18632/oncotarget.13832

Conflict of Interest: The authors declare that the research was conducted in the absence of any commercial or financial relationships that could be construed as a potential conflict of interest.

Copyright $(2021$ Yan, He, Li, Liu and Gao. This is an open-access article distributed under the terms of the Creative Commons Attribution License (CC BY). The use, distribution or reproduction in other forums is permitted, provided the original author(s) and the copyright owner(s) are credited and that the original publication in this journal is cited, in accordance with accepted academic practice. No use, distribution or reproduction is permitted which does not comply with these terms. 